\title{
Herramientas de innovación docente en grupos reducidos: aprendizaje cooperativo y mapas conceptuales \\ Teaching innovation tools in limited groups: cooperative learning and conceptual maps
}

\author{
Josefina Novejarque Civera, Mabel Pisá Bó \\ josefina.novejarque@esic.edu, mabel.pisa@esic.edu \\ Departamento Economía y Finanzas \\ Esic Business Marketing \& School \\ Valencia, España
}

\begin{abstract}
Resumen- En este estudio se analizan los beneficios del uso de un combinado de técnicas docentes, La Tecnica Puzzle Aronson (TPA) y los Mapas Conceptuales (MC), en grupos reducidos de contabilidad financiera. Se han analizado dos grupos durante dos cursos académicos consecutivos; el experimental, en el que se ha aplicado el combinado de técnicas; y el de control, en el que se han aplicado clases magistrales. El objetivo del estudio es contratar empíricamente si los resultados académicos mejoran en el grupo experimental al introducir nuevas metodologías de enseñanza. En este sentido, para evaluar el nivel de aprendizaje se ha realizado una prueba escrita. Los resultados se analizan mediante los estadísticos descriptivos y contrastes paramétricos de diferencia de medias y varianzas. El análisis estadístico demuestra que no existen diferencias significativas en las medias de los resultado obtenidos por los individuos de los grupos experimentales, en los dos cursos académicos, al igual que en los grupos de control. Sin embargo, el grupo experimental mejora su rendimiento medio académico respecto al grupo de control, tanto individual con en su conjunto.
\end{abstract}

Palabras clave: Aprendizaje cooperativo, Mapas conceptuales, Docencia en contabilidad financiera, Resultados de aprendizaje

Abstract- This exploratory study analyzes the benefits of using a mix of different teaching techniques, the Aronson Puzzle Method (APM) and the Conceptual Maps (CM), in limited groups of financial accounting. Two groups have been analyzed during two consecutive academic courses; the experimental one, in which the techniques cocktail has been applied; and that of control, in which lectures (teaching method) have been applied. The target of the study is to hire empirically if the academic results improve in the experimental group on having introduced new education methodologies. In this sense, to evaluate the learning level a written test has been realized. The results are analyzed by means of the descriptive statisticians and contrasts parametric of difference of averages and variances. The statistical analysis demonstrates that significant differences do not exist in the averages of them turned out obtained by the individuals of the experimental groups, in two academic courses, as in the control groups. Nevertheless, the experimental group improves its academic yield with regard to the control group, so much individual with in its set.

Keywords: Cooperative learnig, Conceptual maps, Financial accounting, Learning outcomes

\section{INTRODUCCIÓN}

El proceso de enseñanza-aprendizaje en los estudios universitarios ha recaído tradicionalmente en el profesor y en su capacidad de transmitir conocimientos. Sin embargo, el actual contexto Europeo de Enseñanza Superior (EEES) ha promovido un cambio en el modelo de enseñanza, centrado en el estudiante y en su capacidad de aprendizaje. Ello ha llevado a que en la enseñanza universitaria, las tradicionales metodologías docentes deben complementarse con nuevas metodologías más centradas en los estudiantes.

El aprendizaje cooperativo es una metodología de trabajo en grupo y está basada en la interdependencia positiva y la responsabilidad individual y grupal. El aprendizaje significativo es una metodología que interrelaciona los conocimientos nuevos con los previamente adquiridos. Debido las características de ambas metodologías, su aplicación es adecuada a la asignatura de contabilidad. Así lo demuestra la literatura previa sobre temas de innovación docente en esta área. El trabajo cooperativo es una tipología de trabajo en grupo fundamentada en el descubrimiento de los conocimientos, por parte del estudiante, que son transformados en conceptos reconstruidos y expandidos mediante experiencias de aprendizaje relacionales (Zañartu, 2003).

Bruffe (1995) y Zañartu (2003) coinciden en señalar que el trabajo cooperativo es el más indicado cuando los conocimientos exigen memorización, cálculos y procedimientos matemáticos. Este sistema de trabajo está centrado en el estudiante y controlado por el profesor (Maldonado Pérez, 2007). Así pues, el trabajo cooperativo es el más adecuado a la tipología de asignatura motivo de este estudio, la contabilidad financiera. Ésta precisa; por una parte, memorizar la normativa contable; y por otra, utilizar, en ocasiones, cálculos matemáticos. La responsabilidad en el aprendizaje es asumida por el profesor, y el resultado final es la suma de las subtareas realizadas individualmente y repercutirán en el aprendizaje grupal.

Los componentes básicos de la cooperación son: la interdependencia positiva, la responsabilidad individual y de grupo, la interacción cara a cara, las habilidades interpersonales y de pequeños grupos, y el proceso grupal (Turrión y Ovejero, 
2013). Sin embargo, para poder obtener resultado positivos con el aprendizaje cooperativo son básicas las dos primeras (Slavin, 1995). Así pues, el aprendizaje cooperativo como metodología docente basada en el trabajo en grupo es "un entorno de instrucción en el que los estudiantes trabajan en equipos, compartiendo sus objetivos y la responsabilidad en el aprendizaje de los otros miembros del grupos" (Ravenscroft et al., 1995).

Esta metodología ha sido aplicada en investigaciones relacionadas con la docencia universitaria en contabilidad (Apostolou et al., 2010, 2013, entre otros). Tras realizar una revisión de la literatura, encontramos dos líneas de investigación, que difieren en el objetivo a conseguir. La primera integrada por aquellos estudios cuya finalidad es contrastar empíricamente el apredizaje cooperativo frente a otras técnicas docentes mediante los resultados obtenidos (Clinton y Kohlmeyer, 2005; Gabbin y Wood, 2008; Hwang et al., 2005, 2008; Delgado y Castrillo, 2015). La segunda línea de investigación centra su estudio en contrastar empíricamente el efecto del aprendizaje cooperativo en la actitud del alumnado (Ballatine y Larres, 2007, 2009; Farrell y Farrell, 2009; Mate et al., 2011; García-Benau y Zorio, 2012; Prieto et al., 2014).

El presente estudio puede enmarcarse en la primera línea de investigación, pues pretendemos contratar empíricamente los resultados de aprendizaje cooperativo frente a la técnica de clase magistral. El proceso de aprendizaje en el que los conocimientos nuevos adquieren significado por la interacción con los conocimientos, específicos y relevantes, ya existentes, se denomina aprendizaje significativo. Este proceso se realiza progresivamente, pues los conocimientos adquiridos se van integrando a los ya existentes, enriqueciéndolos y haciéndolos más estables (Moreira, 2005a). Esta metodología educativa no consiste en almacenar mecánica y literalmente la información; es decir, en memorizar conceptos que pueden ser reproducidos a corto plazo y aplicados automáticamente a situaciones conocidas, como ocurre con el aprendizaje mecánico (Moreira, 2013); requiere de una actitud activa y reflexiva por parte de la persona que aprende (Moreira, 2005b; Palmero, 2011).

Una técnica que facilita el aprendizaje significativo, es la elaboración de mapas conceptuales (Moreira, 2010). Éstos pueden definirse como una representación gráfica de las relaciones jerarquizadas entre conceptos generales $y$ específicos de una materia o de un tema, es decir, de un campo de conocimiento (Valero y Vaquerizo, 2009).

Los mapas conceptuales, propuestos por Novack y Gowin (1984), son diagramas que permiten simbolizar el sistema de conocimiento. Se diferencian de otras representaciones gráficas por tres de sus características principales: la jerarquización de conceptos, pues permite presentarlos de manera ordenada los conocimientos, de los generales a los específicos; la selección previa de conceptos, pues se precisa de un resumen de los más significativos; y el impacto visual que se obtiene tras su elaboración, pues muestra la relación entre las principales ideas de manera simple y visual (Ontoria et al., 2001).

El uso de esta técnica puede considerase relevante en el actual Espacio Europeo de Enseñanza Superior (EEES) en el que está inmensa la universidad española, y donde las metodologías docentes tradicionales deben complementarse con nuevas metodologías más activas, con el fin de promover la participación activa.
La incorporación de mapas conceptuales en el proceso de aprendizaje es una técnica utilizada en distintas áreas de la enseñanza universitaria; así queda demostrado en la literatura. Tras realizar una revisión de ésta, podemos encontrar estudios efectuados en el área de ingeniería (Albizu y Fondón, 2007; Valero y Vaquerizo, 2009); y en el área de ciencias sociales (Canay y Cantorna, 2009; García-Benau y Zorio, 2012). Ésta última, en concreto a estudiantes de Licenciatura de Administración y Dirección de Empresas y Diplomatura de Ciencias empresariales, en investigaciones relacionadas con la docencia en contabilidad.

Como señalan Albizu y Fondón (2007), se puede justificar la aplicación de mapas conceptuales desde dos perspectivas, la de los alumnos y la de los docentes. La perspectiva de los alumnos se orienta hacia el aprendizaje, pues les permite desarrollar destrezas para descubrir las relaciones entre los conceptos; y favorece el análisis y trabajo en grupo, fortaleciendo el aprendizaje cooperativo. La perspectiva del docente se orienta hacia la enseñanza, pues les ayuda a organizar y representar los materiales de las asignaturas a impartir en el curso académico; y es útil en el proceso de evaluación de los alumnos, tanto de manera individual como grupal.

Pero los mapas conceptuales deben ser explicados por el profesor, pues no son autoinstructivos. Deben ser utilizados para dar una visión general del tema de estudio, pero es preferible que los alumnos lo utilicen cuando éstos hayan obtenido unos conocimientos previos sobre el tema de estudio (Moreira, 2005b). Así pues, esta técnica puede enmarcarse en nuestro estudio como complemento del aprendizaje cooperativo, pues pretendemos demostrar que los mapas conceptuales ayudan a integrar y diferenciar el significado de los conceptos adquiridos.

El presente estudio tiene por objetivo analizar la confianza de una de las técnicas del aprendizaje cooperativo, la Tecnica Puzzle de Aronson (TPA), combinada con una técnica del aprendizaje significativo, la elaboración de Mapas Conceptuales (MC), en la mejora de los resultados académicos del alumnado. Para ello se contrastarán los resultados obtenidos con esta metodología frente a la metodología tradicional de clase magistral. Después de esta introducción, el epígrafe siguiente está destino al contexto y objetivos del estudio, y público objetivo. El tercer epígrafe explica las metodologías aplicadas, los datos e hipótesis utilizadas. El cuarto epígrafe recoge los resultados empíricos obtenidos. En el último epígrafe se presentan las conclusiones más relevantes del estudio realizado.

\section{ConteXto}

\section{A. Contexto y objetivos}

El presente estudio ha sido realizado en el centro ESIC Valencia durante los cursos académicos 2015-2016 y 20162017, en la asignatura contabilidad financiera. Dicha asignatura se cursa en tres de las cuatro titulaciones oficiales ofertadas por centro, Grado en Administración y Dirección de Empresas (GRADE), Grado en International Business (GRIB) y Grado en Comunicación y Marketing (GRCM). Se trata de una asignatura de formación básica; seis créditos European Credit Transfer System (ECTS o Sistema Europeo de Transferencia de Créditos), que corresponde a cuatro horas lectivas semanales; y se imparte en el segundo semestre de primer curso (en todas las titulaciones). Aunque la asignatura 
de contabilidad financiera se imparte en las tres titulaciones oficiales donde la guía docente es muy similar en objetivos, resultados de aprendizaje, normas de evaluación y programa de la asignatura; la ponderación de la nota de evaluación continua sobre la nota final difiere de una titulación a otra.

En el sistema de evaluación, el peso de la evaluación continua respecto a la nota final es del $15 \%$ en GRADE, del $30 \%$ en GRIB y del 10\% en GRCM. Para poder tener derecho a la misma, se les exige a los alumnos la asistencia obligatoria a clase, estableciendo un porcentaje mínimo de asistencia del 75\%. Pero esta norma sólo es aplicable a los alumnos matriculados por primera vez en dicha asignatura. Los alumnos repetidores, debido a la coincidencia de horarios con otras asignaturas de cursos superiores, las cuales tienen preferencia a la hora del cómputo de asistencia, están exentos de dicha normativa. Para poder optar a la nota de continua, se imparten seminarios en horario no lectivo. Respecto a los alumnos matriculados por primera vez en la asignatura, presentan una falta de asistencia significativa en todos los grupos, de sendos cursos académicos. Esta falta de asistencia varía según titulación, siendo la titulación de GRIB la que presenta menor índice de faltas de asistencia (8\%); y la titulación de GRCM la que mayor porcentaje presenta (45,45\%). Este hecho se debe fundamentalmente, al interés que suscita la nota de continua en dichas titulaciones (30\% y $10 \%$, respectivamente).

Se ha elegido esta asignatura por ser una materia en la que se establecen los fundamentos y aspectos generales de la contabilidad financiera y es común a las tres titulaciones. Por su carácter introductorio a la contabilidad, su contenido resulta bastante arduo y hostil para el alumnado. Este hecho puede verse aumentado, posiblemente, por ser una asignatura que se imparte en el primer curso de la titulación universitaria; en el que los estudiantes se encuentran inmersos en un proceso de adaptación a una nueva etapa educativa, la universidad. Si a estas dos premisas se les suma el bajo índice de asistencia, y comparamos los resultados académicos de esta asignatura con el resto de las que se imparten en el mismo periodo académico, nos encontramos con unos resultados poco satisfactorios. De hecho, es una de las asignaturas que menor índice de aprobados presenta, entre las asignaturas impartidas en primer curso.

La asignatura de contabilidad financiera cuenta con un temario dividido en dos partes, claramente diferenciadas. En la primera parte, se estudiarán los conceptos teóricos básicos de contabilidad financiera. Respecto a la segunda parte, se realizará una aplicación práctica de los conceptos aprendidos en la primera parte y relacionados con la práctica contable, aplicando la normativa contable española el Plan General Contable (PGC).

Adicionalmente, la asignatura está coordinada y es impartida por el mismo profesor, tanto las clases teóricas como prácticas. La metodología docente utilizada en todos los grupos es idéntica, se alternan las clases teóricas y prácticas, sin existir un horario preestablecido para ello. La teoría se imparte mediante clases magistrales, apoyadas con presentaciones en powerpoint (práctica contratada en esta disciplina por Sugahara y Boland, 2006), para cada uno de los temas que conforman la guía docente. Dichos temas están fundamentados en varios manuales a disposición del alumno en la biblioteca del centro. Una vez finalizada la explicación de los contenidos teóricos de cada tema, se plantean y realizan las prácticas (en clase y fuera de ella). Éstas consisten en una colección de supuestos, comunes para ambos grupos, en los que se aplican los conocimientos teóricos adquiridos. Tanto las presentaciones de los temas teóricos como los supuestos prácticos son facilitados a los estudiantes al inicio del curso a través del aula virtual.

El estudio ha sido aplicado en la segunda parte del temario, concretamente en el tema relacionado con los acreedores y deudores por operaciones comerciales. Las razones que han llevado a elegir este tema son varias, en primer lugar los alumnos ya han adquirido los conocimientos suficientes para poder entender y aplicar la normativa contable relacionada con este tema. En segundo lugar, el profesor ha tenido suficiente tiempo para poder conocer los grupos de alumnos y decidir qué grupo es el más adecuado para aplicar una técnica docente innovadora.

\section{B. Público objetivo}

La población inicial, en el curso académico 2015-2016, estaba formada por tres grupos cuyas características son muy similares en un número de alumnos, concretamente 28 alumnos en GRADE (grupo experimental); 47 alumnos en GRCM y 30 alumnos en GRIB (grupos de control). Si excluimos a los alumnos repetidores, por los motivos explicados anteriormente, la población inicial queda conformada por 22 alumnos en el grupo experimental; y en sendos grupos de control, 34 alumnos en GRCM, y 28 alumnos en GRIB. Como consecuencia de ello, no existe desdoblamiento en las clases prácticas.

Esta muestra se vio nuevamente reducida debido a la falta de asistencia de algunos alumnos matriculados por primera vez en la asignatura, y con ello el abandono de la misma. Concretamente el número de alumnos matriculados por primera vez en la asignatura y que la han abandonado es de 4 en el grupo experimental GRADE (el 18,2\%); 11 alumnos en el grupo de control GRCM (33,3\%); y 6 alumnos en el grupo de control GRIB (21,43\%). Como consecuencia de ello, la muestra final, del curso académico 2015-2016, quedo constituida por 18 alumnos, en el grupo de control; y por 22 y 23 alumnos, respectivamente, en sendos grupos experimentales.

Respecto al curso académico 2016-2017, se estableció como grupo experimental la titulación de GRIB y como grupos de control las titulaciones de GRADE y GRCM. La población inicial de este curso académico estaba conformada por 33 alumnos en el grupo experimental y por 29 y 58 alumnos en los respectivos grupos experimentales.

Al igual que se realizó en la población del curso académico 2015-2016, en el presente curso académico se eliminaron los alumnos repetidores. Concretamente, 8 alumnos en GRIB, 9 alumnos en GRADE y 14 en GRCM. La muestra final quedo reducida y únicamente conformada por los alumnos de primera matricula, 33 alumnos en el grupo experimental; y por 20 y 44 alumnos en los grupos de control, respectivamente.

Pero esta muestra se vio nuevamente reducida, al igual que el año anterior, por la falta de asistencia de algunos alumnos. El grupo experimental se vio reducida en un 8\% (2 alumnos); y los grupos de control, GRADE y GRCM, en un 20\% (4 alumnos) y un 45,5\% (20 alumnos), respectivamente. Así pues, la muestra del actual curso académico quedo conformada por 23 alumnos en el grupo experimental, 16 alumnos en GRADE y 24 alumnos en GRCM. 
Para evaluar los conocimientos adquiridos en el tema sujeto a estudio, se ha realizado una prueba escrita que formaba parte de la nota de continua. Esta prueba consistía en un ejercicio práctico de varios asientos relacionados con las distintas transacciones económicas relacionadas con los deudores comerciales.

Con los datos obtenidos en la prueba escrita se pretenden contrastar las siguientes hipótesis:

H1: Las nuevas técnicas de innovación docente, concretamente el uso de la Técnica del Puzzle de Aronson completado con la elaboración de Mapas Conceptuales permite no diferenciar los resultados académicos en grupos reducidos de contabilidad con independencia de la titulación y curso académico.

H2: Las clases magistrales como técnica docente permite no diferenciar los resultados académicos en grupos reducidos de contabilidad con independencia de la titulación y curso académico.

H3: La utilización de nuevas técnicas de innovación docente, concretamente el uso de la Técnica del Puzzle de Aronson completado con la elaboración de Mapas Conceptuales frente a la clase magistral mejora el rendimiento medio académico de los alumnos.

Esta hipótesis será contrastada mediante estadísticos descriptivos y contraste de diferencia de medias, estadístico tStudent, para muestras independientes.

\section{DESCRIPCIÓN}

\section{A. Metodología, datos e hipótesis}

La metodología aplicada en el estudio es una combinación de la Técnica Puzzle de Aronson (TPA) complementada con la elaboración de Mapas Conceptuales (MC).

Esta técnica consiste básicamente en dividir a los alumnos en grupos de trabajo, denominados grupos originales o nodriza, y a cada componente del grupo (al que denominaremos experto) se le asignará y responsabilizará de la preparación de un epígrafe del tema a tratar. Posteriormente, cada uno de los expertos de los grupos nodriza se reúnen (grupos de expertos) para debatir sobre la parte del tema asignada. Una vez finalizada la reunión de expertos, en la que se han perfilado y resuelto todos los aspectos relacionados con el epígrafe asignado, regresan a su grupo nodriza y explican al resto del grupo lo que han estado preparando; convirtiéndose así en profesor de una parte y alumno del resto, dentro de un tema de estudio.

Una vez finalizada la sesión del puzzle y con los conocimientos adquiridos durante la misma, los alumnos completaron un mapa conceptual, elaborado por el profesor, donde se interrelacionan los conocimientos adquiridos. Dicha metodología puede definirse como la representación gráfica que se organiza formando jerarquías, que indican las relaciones entre conceptos, o entre palabras, y que son utilizados para la representación de conceptos (Novak, 1998; Moreira, 2005a; Valero y Vaquerizo, 2009).

Para la preparación, desarrollo y elaboración de ambas técnicas, la TPA combinada con MC, los alumnos se han documentado y preparado con ayuda de los apuntes disponibles en el aula virtual, el PGC y distintos manuales de referencia que figuran en la bibliografía de la guía docente.

\section{RESUltados}

Los resultados obtenidos en los estadísticos descriptivos, correspondientes a las notas (valorada sobre 10 puntos) de la prueba escrita obtenidas por los grupos experimentales en los dos cursos académicos 2015-2016-GRADE (grupo 1) y 20162017-GRIB (grupo 2), se muestran en la tabla 1. Como se puede observar la media y la mediana del grupo $1(6,42 \mathrm{y}$ 6,92) son superiores a la de grupo 2 (5,58 y 5,83). La dispersión de las notas respecto de la media en el grupo 1 $(1,77)$ es menor que en el grupo $2(1,89)$. Así pues, los resultados obtenidos en sendos grupos experimentales presentan diferencias en la media, mediana y desviación típica poco significativa y como consecuencia de ello, podemos decir que son similares.

Tabla 1. Estadísticos descriptivos grupos experimentales

\begin{tabular}{|l|l|l|l|c|c|c|}
\hline Grupo - & $\mathbf{N}$ & $\mathbf{m}$ & $\mathbf{M}$ & Media & Med & Desv. tip. \\
\hline Grupo 1 & 18 & 0,76 & 8,50 & 6,42 & 6,92 & 1,77 \\
Grupo 2 & 23 & 1,67 & 9,17 & 5,58 & 5,83 & 1,89 \\
\hline
\end{tabular}

En relación a los resultados obtenidos en el contraste de diferencia de medias para muestras independientes, prueba tStutend, la variable metodológica ofrece una significatividad inferior al 5\% (véase tabla 2). La diferencia de notas entre los dos grupos no es significativa, como ya se apreciaba en los estadísticos descriptivos.

Tabla 2. Prueba de t- Student para muestras independientes grupos experimentales

\begin{tabular}{|l|c|c|}
\hline Grupo & t-Student & Sig. asintót. (bilatl) \\
\hline Grupo1-Grupo2 & $-1,973$ & $0,0558^{* *}$ \\
\hline
\end{tabular}

Vistos los resultados obtenidos en los estadísticos descriptivos y en la prueba de diferencia de media, podemos concluir que aceptamos la hipótesis nula H1: Las nuevas técnicas de innovación docente, concretamente el uso de la Técnica del Puzzle de Aronson completado con la elaboración de Mapas Conceptuales permite no diferenciar los resultados académicos en grupos reducidos de contabilidad con independencia de la titulación y curso académico.

Respecto a los resultados obtenidos en los grupos experimentales: GRCM 15-16 (grupo 3), GRIB 15-16 (grupo 4), GRADE 16-17 (grupo 5) y GRCM 16-17 (grupo 6)), los estadísticos descriptivos son los que figuran en la tabla 3. Como se puede apreciar, las medias de los grupos 3 y 4 (5,46 y 5,71) son similares; al igual que ocurre con las medias de los grupos 5 y 6 (3,52 y 3,88). En relación a la desviación típica de los cuatros grupos, todas ellas se encuentran entre 2,03 (grupo 3) y 3,49 (grupo 5).

Tabla 3. Estadísticos descriptivos grupos de control

\begin{tabular}{|l|l|l|r|r|r|r|}
\hline Grupo & N & $\mathbf{m}$ & \multicolumn{1}{l|}{ M } & Media & Med & Desv. tip. \\
\hline Grupo 3 & 23 & 0,77 & 8,46 & 5,46 & 5,38 & 2,03 \\
Grupo 4 & 22 & 0,71 & 10,00 & 5,71 & 5,55 & 2,70 \\
Grupo 5 & 16 & 0,00 & 10,00 & 3,52 & 2,08 & 3,49 \\
Grupo 6 & 24 & 0,00 & 9,17 & 3,88 & 3,33 & 3,29 \\
\hline
\end{tabular}


La tabla 4 muestra los resultados obtenidos, para los grupos de control, en el contraste de diferencia de medias para muestras independientes, con una significatividad inferior al $5 \%$. El valor obtenido para dichos grupos demuestra que la diferencia de medias entre los dos grupos no es significativa.

Tabla 4. Prueba de t-Student para muestras independientes grupos de control

\begin{tabular}{|l|c|c|}
\hline Grupo & t-Student & Sig. asintót. (bilateral) \\
\hline Grupo 3-Grupo 4 & 0,344 & $0,7326^{* *}$ \\
Grupo 3-Grupo 5 & 2,000 & $0,0579^{* *}$ \\
Grupo 3-Grupo 6 & 1,711 & $0,1011^{* *}$ \\
Grupo 4-Grupo 5 & 2,176 & $0,0362^{*}$ \\
Grupo 4-Grupo 6 & 1,878 & $0,0685^{* *}$ \\
Grupo 5-Grupo 6 & 0,297 & $0,7985^{* *}$ \\
\hline
\end{tabular}

Los resultados obtenidos para los grupos de control, estadísticos descriptivos y pruebas paramétricas, permiten aceptar la hipótesis nula H2: Las clases magistrales como técnica docente permite no diferenciar los resultados académicos en grupos reducidos de contabilidad con independencia de la titulación y curso académico.

Una vez realizados los contrastes estadísticos oportunos, que nos permiten aceptar las hipótesis $\mathrm{H} 1$ y H2, juntamos los dos grupos experimentales y los cuatros grupos de control. La muestra experimental queda ahora conformada por 41 observaciones; y la de control, por 85 observaciones.

Los estadísticos descriptivos, correspondientes a las nuevas muestras (tabla 5), presentan una media superior a 1,08 en el grupo experimental respecto al grupo de control. La medina también es superior en el grupo experimental $(6,20)$ respecto al de control $(5,38)$. La desviación típica en el grupo de control $(1,85)$ es menor a la del grupo de control $(2,94)$.

Tabla 5. Estadísticos descriptivos grupo experimental y de control

\begin{tabular}{|l|l|l|l|l|l|l|}
\hline Grupo & N & m & M & Media & Med & $\begin{array}{l}\text { Desv. } \\
\text { tip. }\end{array}$ \\
\hline $\begin{array}{l}\text { Experi } \\
\text { Control }\end{array}$ & 41 & 0,77 & 9,17 & 5,96 & 6,20 & 1,85 \\
\hline
\end{tabular}

La prueba t-Student presenta un valor de 2,153 con una significatividad del 5\% (véase tabla 6). El valor obtenido permite concluir que la media para ambos grupos es distinta. Como ya se observaba en los estadísticos descriptivos. la media del grupo experimental es superior a la del grupo de control.

Tabla 6. Prueba de t de Student para muestras independientes grupos experimental y de control

\begin{tabular}{|l|l|l|}
\hline Grupo & t-Student & Sig. asintót. (bilateral) \\
\hline Experi - Control & 2,153 & $0,0166^{* *}$ \\
\hline
\end{tabular}

Vistos los resultados obtenidos en los estadísticos descriptivos y en la prueba de diferencia de medias, podemos concluir que aceptamos la hipótesis nula H3: La utilización de nuevas técnicas de innovación docente, concretamente el uso de la Tecnica del Puzzle de Aronson completado con la elaboración de Mapas Conceptuales frente a la clase magistral mejora el rendimiento medio académico de los alumnos.

\section{CONCLUSIONES}

El actual marco normativo universitario se caracteriza por un cambio en el modelo de enseñanza. La innovación docente y las nuevas metodologías de enseñanza-aprendizaje se están estableciendo en el ámbito de las distintas áreas de la docencia universitaria desde hace años. La contabilidad no ha estado exenta de esta influencia, y por ello, cada vez más se están instaurando nuevas metodología en la enseñanza de la misma.

Un análisis de la literatura internacional revela que cada vez más se publican estudios que demuestran empíricamente el efecto que las nuevas metodologías docentes tienen sobre los resultados académicos, suponiendo un nuevo aliciente frente a la clase magistral.

El aprendizaje cooperativo es una metodología docente relacionada con la enseñanza universitaria utilizada en la asignatura de contabilidad. Los resultados de aprendizaje frente a otras técnicas docentes, hacen de esta técnica una de las más adecuadas para la contabilidad. Dicha metodología se caracteriza, principalmente, por la interdependencia positiva y la responsabilidad individual y grupal. Una de las técnicas de este aprendizaje es la TPA. Entre sus principales ventajas se encuentran la interacción entre los alumnos, además de promover el autoaprendizaje.

Otra metodología docente utilizada en el ámbito de la enseñanza universitaria en la asignatura de contabilidad es el aprendizaje significativo. Éste se caracteriza por consolidar el conocimiento previo y adquirir significado el nuevo conocimiento. Una de las técnicas que facilitan este tipo de aprendizaje son los mapas conceptuales. Estos son diagramas que indican relaciones entre conceptos y estructuran el conocimiento. Para ello, el alumno debe presentar una predisposición al aprendizaje.

En esta comunicación, que presentamos en el IV CINAIC, hemos realizado un estudio de metodologías docentes combinadas. Con éste se pretende demostrar el combinado de la TPA con los MC tiene un efecto positivo sobre el rendimiento académico en un grupo reducido de contabilidad financiera en los cursos académicos 2015-2016 y 2016-2017. Los resultados obtenidos con los grupos experimentales de control se han contrastado empíricamente, los estadísticos descriptivos y el contraste de medias de las notas obtenidas por ambos grupos corroboran que combinar la TPA con los MC es una metodología docente que mejora los resultados académicos del alumnado. Este estudio ha servido para confirmar las ventajas de la aplicación de nuevas metodologías docentes en el ámbito de 1 a enseñanza universitaria; y esperamos que sirva de preámbulo para futuros trabajos de investigación.

\section{REFERENCIAS}

Abizu, M. R. y Fondón, M. A. (2007). La revisión entre iguales como herramienta de aprendizaje y evaluación en la asignatura de sistemas operativos. Barcelona: XIII Jornadas sobre Enseñanza Universitaria de la Informática (JENUI). [consultado 14 de marzo de 2017]. Disponible en: http://di002.edv.uniovi.es/. 
Apostolou, B., Dorminey, J. W., Hassell, J. M. y Watson, S. F. (2013). Accounting education literature review (20102012). Journal of Accounting Education, 31(2), pp.107161.

Apostolou, B., Hassell, J. M., Revele, J. E. y Watson, S. F. (2010). Accounting education literature review (20062009). Journal of Accounting Education, 28(3-4), pp.145-197.

Ballantine, J. y Larres, P. M. (2007). Cooperative learning: a pedagogy to improve student' generic skills? Education and Training, 49(2), pp. 126-137.

Ballantine, J. y Larres, P. M. (2009). Accounting undergraduates perceptions of cooperative learning as a model for enhancing their interpersonal and communication skill to interface successfully with professional accountancy education and training. Accounting Education: An International Journal, 18(45), pp.387-402.

Bruffee, K.A. (1995). Sharing our toys. Cooperative learning versus collaborative learning. Change, Jan/Feb, pp. 1218

Canay Pazos, J.R. y Cantorna Agra,S. (2009). Los mapas conceptuales en contabilidad: una herramienta para el EEES. Sevilla: VI Jornada de Docencia en Contabilidad ASEPUC [consultado 14 de marzo de 2017]. Disponible en: http://hdl.handle.net/10317/2109

Clinton, B. D. y Kohlmeyer, J. M. (2005). The effects of group quizzes on performance and motivation to learn: Two experiments in cooperative learning. Journal of Accounting Education, 23(2), pp. 96-116.

Delgado Hurtado, M.M., Castillo Lara, L.A. (2015). Revista de Contabilidad - Spanish Accounting Review 18 (2), pp. 138-147.

Farrell, B. J. y Farrell, H. M. (2009). Student satisfaction with cooperative learning in an accounting curriculum. Journal of University Teaching and Learning Practice, 5(2), pp. 39-54.

Gabbin, A. L. y Wood, L. I. (2008). An experimental study of accounting majors academic achievement using cooperative learning groups. Issues in Accounting Education, 23(3), pp. 391-404.

García-Benau, M.A. y Zorio Grima, A. (2012). Experiencia sobre la utilización de un mix de metodologías docentes en la educación universitaria de la contabilidad. Cuadernos de Contabilidad, 13 (33), pp. 613-657.

Hwang, N. R., Lui, G. y Tong, M. Y. (2008). Cooperative learning in a passive learning environment: A replication and extension. Issues in Accounting Education, 23(1), pp. 67-75.

Hwang, N. R., Lui, G. y Tong, M. Y. J. W. (2005). An empirical test of cooperative learning in a passive learning environment. Issues in Accounting Education, 20(2), pp. 151-165.

Maldonado Pérez, M. (2007). El trabajo colaborativo en el aula universitaria. Laurus, 13(23), 263-278 [consultado 4 de febrero de 2017]. Disponible en: http://www.redalyc.org/articulo.oa?id=76102314
Maté, M. L., Madrid, A. y García, E. (2011). Técnicas de aprendizaje cooperativo en la docencia de análisis de los estados financieros. Palma de Mallorca: VIII Jornada de Docencia en Contabilidad ASEPUC [consultado 26 de marzo de 2017]. Disponible en: http://hdl.handle.net/10317/2109

Moreira, M. A. (2005a). Aprendizaje significativo critico. Indivisa: Boletín de estudios e investigación, 6, pp. 83101.

Moreira, M. A. (2005b). Mapas conceptuales y aprendizaje significativo en ciencias. http:/innovaeduc.files.wordpress.com/2008/04/mapasesp .pdf, adaptado y acualizado de un trabajo previo publicado en Cadernos do Aplicacao, Porto Alegre 11 (2), 1998.

Moreira, M.A. (2010), Mapas conceituais e aprendizagem significativa, Sao Paulo: Centauro Editora, 80p

Moreira, M.A. (2013). Aprendizaje significativo en mapas conceptuales. Meaningful Learning Review, 3 (2), pp. 35-76

Novak, J. D. y Gowin, D. B. (1998). Aprendiendo a aprender. Barcelona: Martínez Roca.

Novak, J. D. y Gowin, D. B. (1998). Learning how to learn. New York. Cambridge Iniversity Press.

Ontoria Peña, A. et al (2001). Mapas Conceptuales: Una técnica para aprender. Narcea, Madrid-

Turrión Borrallo, P. y Ovejero Bernal, A. (2013). ¿Es eficaz el aprendizaje cooperativo para la mejora del rendimiento académico en la enseñanza del inglés? Estudio experimental en alumnos de primaria. Tabenque Revista pedagógica, 26, pp. 249-266

Palmero, M. L. R. (2011). La teoría del aprendizaje significativo: una revisión aplicable a la escuela actual. IN. Investigació i Innovació Educativa i Socioeducativa, 3(1), pp. 29-50.

Prieto et al., (2014). Cooperative learning in the Zone of Proximal Development: an experience in the Secondary Education master's. International Journal of Educational Research and Innovation, 3, pp. 64-77.

Ravenscroft, S., Buckles, F. y Zuckerman, G. (1995). Incentives in student learning: an experiment in cooperative learning. Issues in Accounting Education, 10(1), pp.97-109.

Slavin, R.E. (1995). A model of effective instruction. The Educational Forum, 59, pp.166-176.

Sugahara, S. and Boland, G, (2006). The Effectiveness of PowerPoint presentations in the Accounting Classroom. Accounting Education, 15 (4), pp.391-403.

Zañartu Correa., L. M. (2003). Aprendizaje colaborativo: una nueva forma de Diálogo Interpersonal y en Red. Revista Digital de Educación y Nuevas Tecnologías, 28 (V) [consultado 24 de enero de 2017]. Disponible en: http://contexto-educativo.com.ar/2003/4/nota-02.htm 